Ranking Income Distributions

Author(s): Anthony F. Shorrocks

Source: Economica, New Series, Vol. 50, No. 197, (Feb., 1983), pp. 3-17

Published by: Blackwell Publishing on behalf of The London School of Economics and Political

Science and The Suntory and Toyota International Centres for Economics and Related

Disciplines

Stable URL: http://www.jstor.org/stable/2554117

Accessed: 11/08/2008 08:56

Your use of the JSTOR archive indicates your acceptance of JSTOR's Terms and Conditions of Use, available at http://www.jstor.org/page/info/about/policies/terms.jsp. JSTOR's Terms and Conditions of Use provides, in part, that unless you have obtained prior permission, you may not download an entire issue of a journal or multiple copies of articles, and you may use content in the JSTOR archive only for your personal, non-commercial use.

Please contact the publisher regarding any further use of this work. Publisher contact information may be obtained at http://www.jstor.org/action/showPublisher?publisherCode=black.

Each copy of any part of a JSTOR transmission must contain the same copyright notice that appears on the screen or printed page of such transmission.

JSTOR is a not-for-profit organization founded in 1995 to build trusted digital archives for scholarship. We work with the scholarly community to preserve their work and the materials they rely upon, and to build a common research platform that promotes the discovery and use of these resources. For more information about JSTOR, please contact support@ jstor.org. 


\title{
Ranking Income Distributions
}

\author{
By Anthony F. ShORROCKS \\ The London School of Economics and Queen's University, Ontario
}

\section{INTRODUCTION}

A large variety of policy questions involve choices between social states and a consequent ordering of the feasible alternatives. When these social states are related to the levels of welfare experienced by individuals or households, two central issues stand out in determining the relative desirability of different social outcomes. One of these is the essentially positive exercise of achieving comparability between households with different characteristics (such as composition or preferences) operating in different environments (for example, facing different price structures). The other concerns the normative judgments implicit in the evaluation of alternative allocations of resourcesthe emphasis placed on inequality between households and the extent to which greater inequality can be compensated by higher average living standards. This paper focuses on the second of these issues, and in doing so we abstract from the problem of household comparability by considering a population of $n$ households, identical in all respects except for their incomes. ${ }^{1}$ The question of ordering social states then becomes one of ranking income distributions over a group of anonymous households or individuals.

Borrowing the usual assumptions imposed on consumer preferences, we may suppose that a social ordering of income distributions can be represented by a "welfare function",

$$
W=W(\mathbf{y})=W\left(y_{1}, \ldots, y_{n}\right)
$$

where $y_{i}$ is the income of individual $i$. If $W($.$) can be precisely specified, the$ ranking of distributions degenerates into a trivial exercise. But there are few who would insist, without qualification, on one particular functional form for $W($.). The most that might reasonably be assumed is that generally acceptable restrictions can be placed on $W($. ) to allow a partial ordering to be obtained. This paper considers two types of constraints, reflecting a social preference for a more equitable distribution and higher (real) incomes, ceteris paribus. These properties are called "equity preference" and "efficiency preference", respectively. As regards the concept of equity preference, there is a standard definition that is employed throughout the paper. But the notion of efficiency preference is open to a variety of interpretations. We examine a number of the alternative formulations and derive operational rules that would enable the corresponding partial orderings over distributions to be applied empirically.

It is perhaps an exaggeration to claim that there is a standard procedure for ranking distributions. But it is common practice to divide the process into two stages, first comparing the degree of inequality within each distribution and then introducing information on mean incomes. During the first stage it is frequently difficult to make a positive assessment-intersecting Lorenz 
curves are usually seen as sufficient grounds for regarding a pairwise ranking as ambiguous. Even if this stage produces a clear result, the more equal distribution will often have a lower mean, so the overall ranking is again ambiguous, unless one is prepared to specify the appropriate trade-off between equity and efficiency. For these reasons, there has grown up a general pessimism concerning the ability to rank most pairs of distributions and a belief that the issue is predominantly one of personal value judgments. ${ }^{3}$

Making a distinction between the distributional and efficiency aspects of alternative allocations, and viewing the ranking procedure as a two-stage process, may be a helpful analytical device. But the derived ordering is inconclusive for many pairs of distributions that can be ranked under the usual assumptions imposed on welfare functions. Section I examines an ordering that should have a wide degree of support and shows how the ordering can be implemented, by seeking a dominance relation between "generalized Lorenz curves". An application to comparisons between countries in Section II reveals that this ordering ranks successfully in 84 per cent of the cases. This suggests that the general pessimism concerning the ability to rank distributions is unwarranted, even when allowance is made for considerable variation in individual perceptions of inequality and in the emphasis placed on inequality relative to higher incomes.

These conclusions are qualified to some extent in Section III, where we examine weaker interpretations of efficiency preference that may be required if, for instance, the level of satisfaction experienced by individuals is influenced by the standard of living attained by other members of society. The weaker statements of efficiency preference make it progressively more difficult to achieve conclusive distributional rankings until, under the weakest interpretation considered, the corresponding partial ordering becomes more or less useless for practical purposes. A short summary of the paper and its principal conclusions is contained in Section IV.

\section{Partial Orderings of Income Distributions}

We initially consider income distributions defined over a fixed population of $n$ persons, identical in all respects except for their incomes. The possible social orderings are then represented by different choices of symmetric welfare functions $W($.$) . The presumption that society favours a more equitable$ distribution, ceteris paribus, is captured in the usual way by requiring that mean-preserving regressive transfers do not increase welfare. This interpretation of equity preference is equivalent to the assumption that $W($.$) is Schur-$ concave (see Dasgupta et al., 1973)

$$
\text { (S-concavity) } W(\mathbf{B y}) \geq W(\mathbf{y}) \text { for all bistochastic matrices } \mathbf{B} \text {. }
$$

Note that S-concavity implies that

$$
W(\boldsymbol{\Pi} \mathbf{y})=W(\mathbf{y}) \text { for all permutation matrices } \boldsymbol{\Pi}
$$

and hence incorporates the symmetrical treatment of households.

We are interested in situations where one distribution $\mathbf{y}$ can be shown to be at least as desirable as another distribution $\mathbf{y}^{\prime}$ for any choice of welfare function satisfying (2). It is well known that such situations arise when the 
two distributions have identical means ${ }^{4}$ (denoted here by $\left.\mu, \mu^{\prime}\right)$ and nonintersecting Lorenz curves. We will let $L(\mathbf{y}, p), p \in[0,1]$ represent the Lorenz curve corresponding to the distribution $\mathbf{y}$. Then for a finite population of individuals, indexed by their income rank so that $y_{1} \leq y_{2} \leq \cdots \leq y_{n}$,

$$
L(\mathbf{y}, k / n)=\sum_{i=1}^{k} y_{i} / n \mu \quad k=1, \ldots, n
$$

and we follow the usual procedure of defining $L(\mathbf{y}, 0)=0$ and joining up adjacent points of the form $\{k / n, L(\mathbf{y}, k / n)\}$ with straight line segments to complete the Lorenz curve.

Lemma 1 (Dasgupta et al., 1973, Theorem 1; Rothschild and Stiglitz, 1973, Theorem I)

$$
\begin{gathered}
\text { If } \mu=\mu^{\prime} \text {, then } W(\mathbf{y}) \geq W\left(\mathbf{y}^{\prime}\right) \quad \text { for all S-concave } W(.) \\
\text { iff } L(\mathbf{y}, p) \geq L\left(\mathbf{y}^{\prime}, p\right) \quad \text { for all } p .
\end{gathered}
$$

For the concept of "efficiency preference" we wish to capture the desire for higher incomes over lower incomes, and an obvious method of doing so is to require that $W($.$) is a non-decreasing function of all incomes:$

$$
\text { (monotonicity) } W\left(y_{1}, \ldots, y_{n}\right) \text { is non-decreasing in } y_{i} \quad i=1, \ldots, n \text {. }
$$

This condition will be satisfied if the welfare function has the utilitarian form

$$
W(\mathbf{y})=\sum_{i=1}^{n} U\left(y_{i}\right)
$$

where $U($.$) is increasing and concave. More generally, (5) will be satisfied$ whenever the welfare function is Paretian and the utility levels of each individual depend only on their own incomes.

The introduction of a statement of efficiency preference enables us to compare distributions with different means. We will let $\mathscr{W}_{1}$ denote the set of non-decreasing S-concave welfare functions and write $\mathbf{y} \geqslant_{1} \mathbf{y}^{\prime}$ if $\mathbf{y}$ is never inferior to $\mathbf{y}^{\prime}$.

$$
\mathbf{y} \geqslant_{1} \mathbf{y}^{\prime} \quad \text { iff } W(\mathbf{y}) \geq W\left(\mathbf{y}^{\prime}\right) \text { for all } W(.) \in \mathscr{W}_{1} .
$$

A sufficient condition for this to hold is that $\mathbf{y}$ has both a higher mean and higher Lorenz curve than $\mathbf{y}^{\prime}$. For by proportionately raising all incomes in $\mathbf{y}^{\prime}$ until $\mu^{\prime}=\mu$, the level of welfare increases; but the Lorenz curve remains unchanged, so by Lemma 1 the resulting distribution is still not superior to y. Thus

Theorem 1. $\mathbf{y} \geqslant \mathbf{y}^{\prime}$ if $\mu \geq \mu^{\prime}$ and $L(\mathbf{y}, p) \geq L\left(\mathbf{y}^{\prime}, p\right)$ for all $p$.

This result validates to some extent the common practice of separately comparing the means and Lorenz curves of distributions. But the sufficiency conditions are unnecessarily strong and tend to obscure many important situations in which distributions can be ranked. Conditions equivalent to the ordering $\geqslant_{1}$ are given below in Theorem 2 . Similar conditions have been used by Rothschild and Stiglitz (1973), Blackorby and Donaldson (1977) and Willig (1981), ${ }^{5}$ but they do not appear to be widely known or applied empirically. This may be because they have not been interpreted in a way that is simple 
to understand. Yet this is easily accomplished by introducing the notion of a "generalized Lorenz curve" $G L(\mathbf{y}, p)$, constructed by scaling up the ordinary Lorenz curve by the mean of the distribution. Thus

$$
G L(\mathbf{y}, p)=\mu L(\mathbf{y}, p)
$$

and

$$
G L(\mathbf{y}, k / n)=\frac{1}{n} \sum_{i=1}^{k} y_{i} \quad k=1,2, \ldots, n
$$

when $\mathbf{y}$ is ordered in terms of increasing incomes. The relationship between ordinary and generalized Lorenz curves is illustrated in Figure 1.6 We now demonstrate the equivalence between the ordering $\geqslant_{1}$ and ranking distributions on the basis of their generalized Lorenz curves.

\section{Theorem 2}

$$
W(\mathbf{y}) \geq W\left(\mathbf{y}^{\prime}\right) \quad \text { for all } W(.) \in \mathscr{W}_{1} \quad \text { iff } G L(\mathbf{y}, p) \geq G L\left(\mathbf{y}^{\prime}, p\right) \text { for all } p .
$$

Proof. (if) Define $\mathbf{y}^{\prime \prime}$ such that $y_{i}^{\prime \prime}=y_{i}^{\prime}, i=1, \ldots, n-1 ; y_{n}^{\prime \prime}=y_{n}^{\prime}+n\left(\mu-\mu^{\prime}\right)$. Then condition (5) ensures that $W\left(\mathbf{y}^{\prime \prime}\right) \geq W\left(\mathbf{y}^{\prime}\right)$ for all $W(.) \in W_{1}$. Furthermore, $\mu=\mu^{\prime \prime}$ and $G L(\mathbf{y}, p) \geq G L\left(\mathbf{y}^{\prime \prime}, p\right)$ for all $p$. Hence $L(\mathbf{y}, p) \geq L\left(\mathbf{y}^{\prime \prime}, p\right)$ for all $p$, and, by Lemma $1, W(\mathbf{y}) \geq W\left(\mathbf{y}^{\prime \prime}\right)$ for all $W(.) \in \mathscr{W}_{1}$.

(only if) Let $\mathbf{y}, \mathbf{y}^{\prime}$ be ordered vectors, indexed in terms of increasing incomes. Define

$$
W_{k}(\mathbf{y})=\sum_{i=1}^{k} y_{i} / n \quad k=1,2, \ldots, n .
$$

Then $W_{k}(.) \in \mathscr{W}_{1}$ for all $k$, and (9) follows immediately from $W_{k}(\mathbf{y}) \geq W_{k}\left(\mathbf{y}^{\prime}\right)$ for all $k$.

Corollary. If $G L(\mathbf{y}, p) \geq G L\left(\mathbf{y}^{\prime}, p\right)$ for all $p$, then

$$
\Sigma_{i} u\left(y_{i}\right) \geq \Sigma_{i} u\left(y_{i}^{\prime}\right)
$$

whenever $u($.$) is increasing and concave. { }^{7}$

Theorem 2 indicates that an unambiguous ranking for all $W(.) \in \mathscr{W}_{1}$ is obtainable if and only if the generalized Lorenz curves do not intersect. Clearly this latter condition will apply if one of the distributions has both a higher mean and higher Lorenz curve. ${ }^{8}$ But it will also be satisfied in other cases if the higher mean is sufficient to offset the lower part of the Lorenz curve. This is likely to be true in many important practical situations, since differences between Lorenz curves tend to be relatively small compared with variations in mean incomes. For instance, a large sample of countries is unlikely to generate Lorenz curves whose ordinates differ by more than a factor of 2 or 3 (except at the very bottom of the distribution), while mean incomes may well vary by a factor of 10 or 20 . Thus, scaling up the Lorenz curves to form the generalized Lorenz curves will often reveal a dominance relationship that is not apparent from an examination of means and Lorenz curves on their own.

The next section illustrates how generalized Lorenz curves may be applied empirically. However, before this is done it is necessary to extend the analysis to populations of varying sizes. For our purposes it is sufficient to imagine 

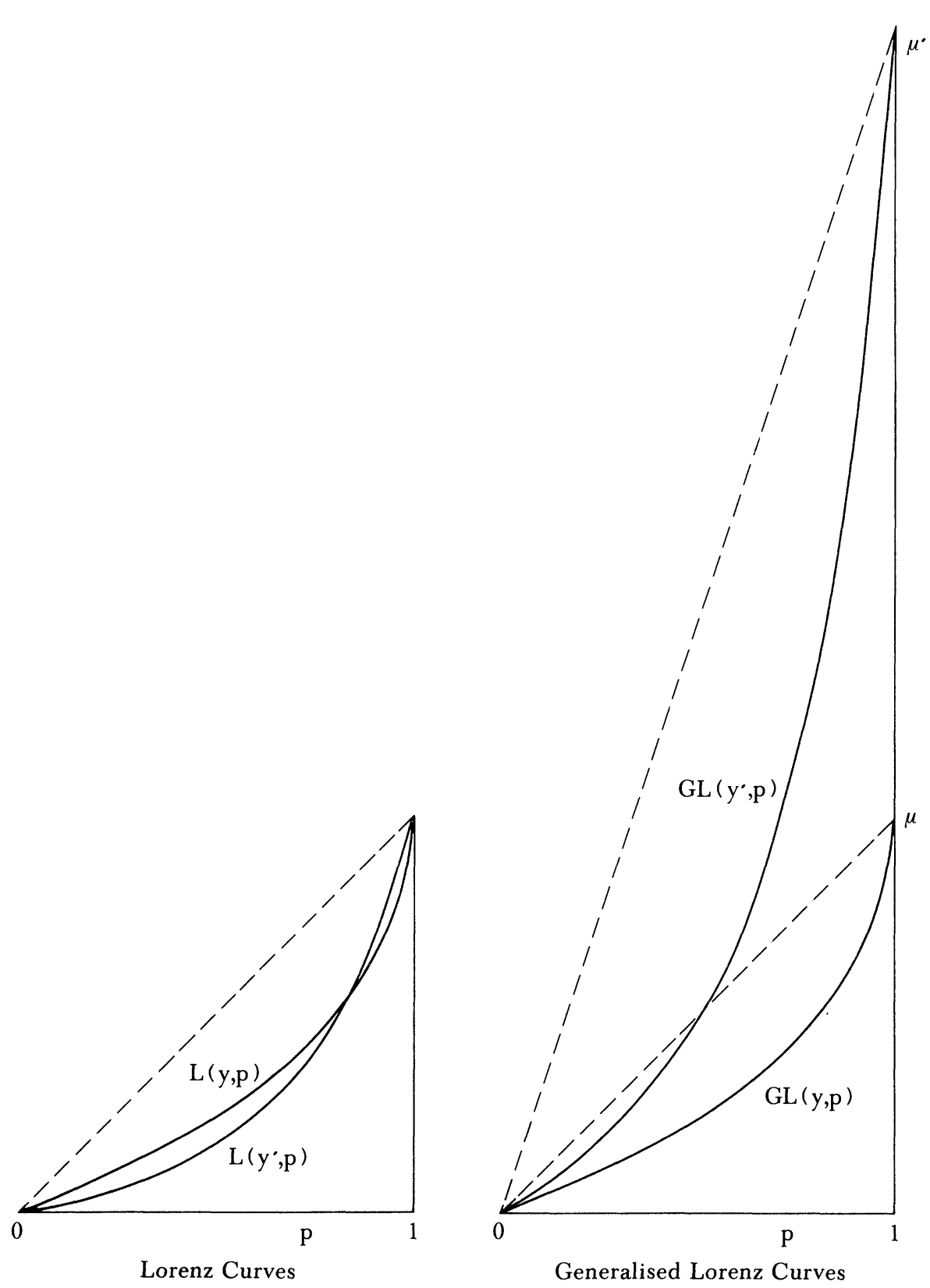

FIGURE 1. (a) Lorenz curves. (b) Generalized Lorenz curves.

that we are examining the "welfare standard" (or "standard of living") of different populations. This allows us to circumvent the issue of whether aggregate welfare tends to rise with larger populations and assume, along with Sen (1976), that $W($.$) is invariant to replication of the population. { }^{9}$

$$
W^{r n}(\mathbf{y}, \mathbf{y}, \ldots, \mathbf{y})=W^{n}(\mathbf{y}) \text { for all positive integers } r
$$

where the superscript is used to indicate the size of the population over which $W($.$) is defined.$ 
Restricting attention to functions satisfying (10) enables Theorem 2 to be extended to communities of different sizes. For if we begin with distributions $\mathbf{y}, \mathbf{y}^{\prime}$ defined over $n, m$ persons, respectively, we can replicate both distributions until we obtain $\hat{\mathbf{y}}, \hat{\mathbf{y}}^{\prime}$ defined over $n m$ persons. Then

$$
W^{n}(\mathbf{y}) \geq W^{m}\left(\mathbf{y}^{\prime}\right) \quad \text { iff } W^{n m}(\hat{\mathbf{y}}) \geq W^{n m}\left(\hat{\mathbf{y}}^{\prime}\right)
$$

and, since population replication leaves the generalized Lorenz curves unchanged,

$$
G L(\mathbf{y}, p) \geq G L\left(\mathbf{y}^{\prime}, p\right) \quad \text { iff } G L(\hat{\mathbf{y}}, p) \geq G L\left(\hat{\mathbf{y}}^{\prime}, p\right) .
$$

\section{An ApplicAtion to Inter-COUntry COMparisons}

To illustrate the problems that frequently arise when comparing distributions and the way that generalized Lorenz curves can help to resolve these problems, we consider an application to distributional data for 20 countries. Figures for cumulated income shares, derived from Jain (1975), are presented in Table 1. These refer to national samples of income recipients and, where a choice is possible, have been selected for the year closest to 1970. Inevitably, the data are likely to be plagued with the usual problems concerning the treatment of casual workers, income recipients below tax or sample thresholds, income from home production and the many other factors that affect comparability across countries. For this reason the precise figures and the ranking obtained should be interpreted with caution. Nevertheless, the inter-country data provide the basis for an interesting application of the results of the previous section.

Inspection of Table 1 reveals that Lorenz curves intersect in at least 108 of the 190 pairwise comparisons between countries. ${ }^{10}$ Thus, barely 40 per cent of the inequality comparisons generate unambiguous rankings. For information on the means of the distributions we use estimates of real purchasing power GDP per capita reported in Kravis et al. (1978). Combining this with the Lorenz curve data shows that, in 29 cases where Lorenz curves do not intersect, the country with the higher Lorenz curve has the lower mean income. So ranking countries only when one has both a higher Lorenz curve and a higher mean would produce conclusive results in just 53 (28 per cent) of the 190 possible pairwise comparisons. The partial ordering obtained is illustrated in Figure 2. ${ }^{11}$

If we set aside for the moment the questions of data reliability and comparability and imagine that we are attempting to assess the desirability of being a random citizen in these countries, the weakness of this ranking becomes apparent. Sweden has over ten times the average income of India, Indonesia, Kenya and Tanzania, but is not ranked above any of these countries because of the lower income share received by the bottom decile of the Swedish population. Yet simple computations reveal that the average income of the bottom 10 per cent, of Swedish income recipients would still place them in the top 5 per cent, and perhaps even the top 1 per cent, of any of these other countries. In these circumstances it is difficult to conceive how the Swedish income distribution could be regarded as inferior to those of India, Indonesia, Kenya or Tanzania. It is this possibility, however, that prevents us 


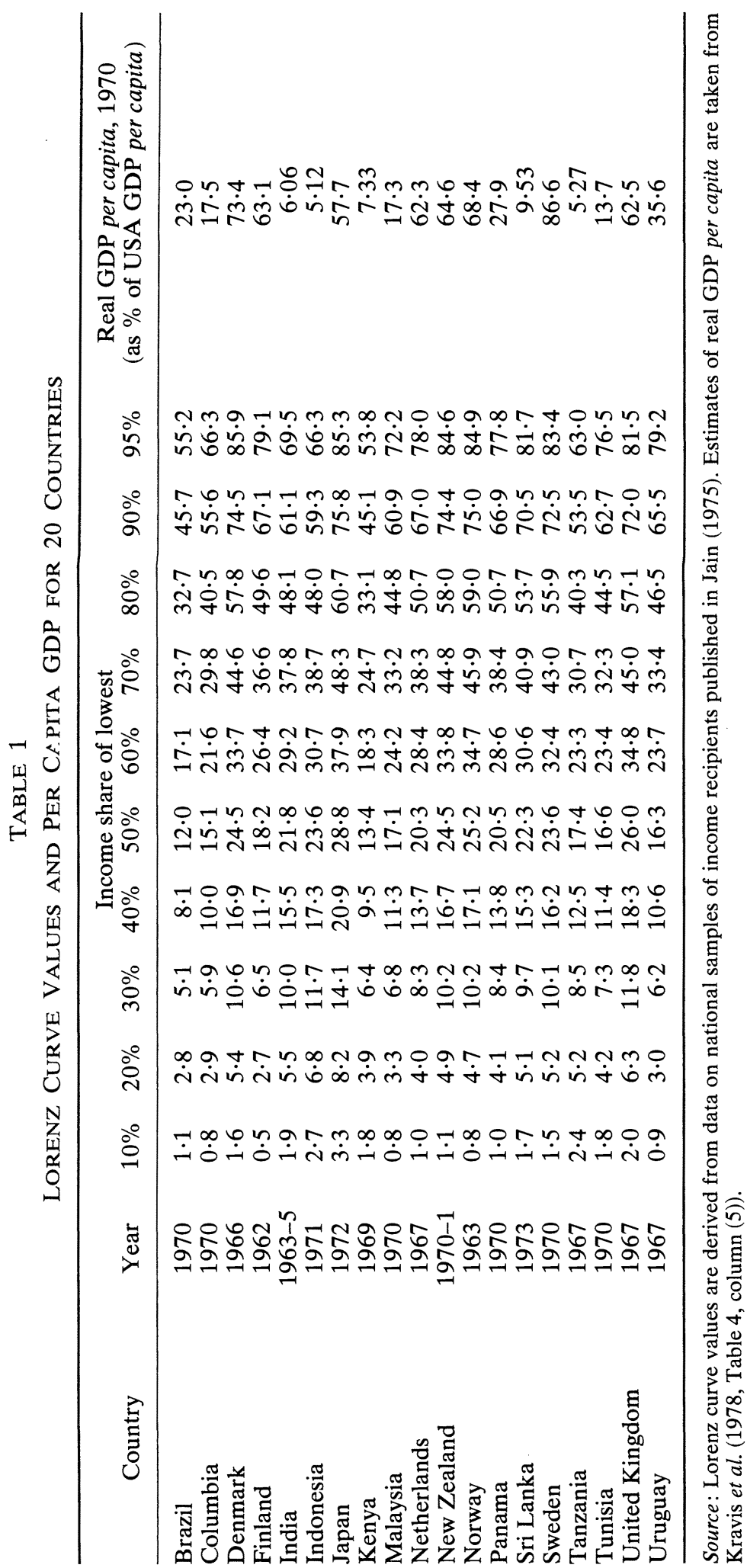


from making a conclusive ranking. Similarly, the United Kingdom cannot be ranked above Sri Lanka simply because the incomes of some of those in the top decile of the UK distribution are relatively lower than their counterparts in Sri Lanka. But their absolute incomes are more than five times greater.

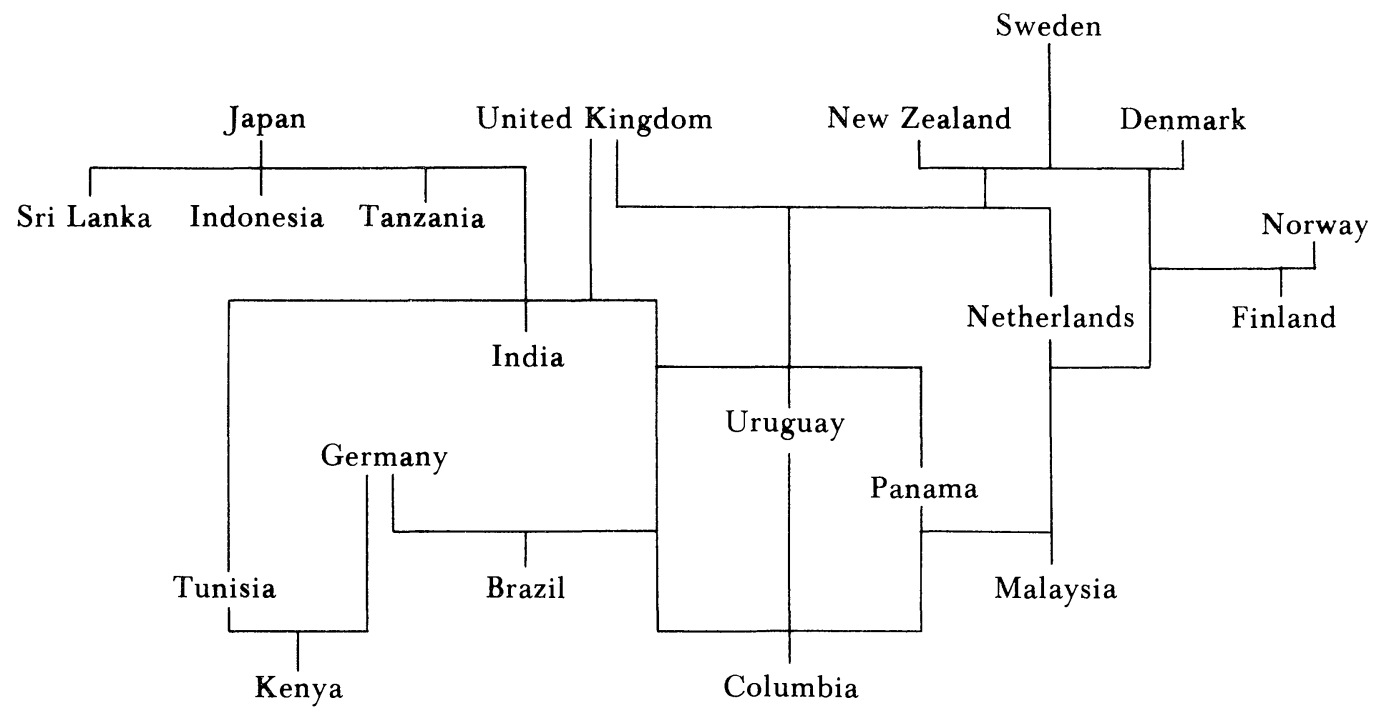

FIGURE 2. Ranking based on higher mean and higher Lorenz curve.

The ordering derived from generalized Lorenz curves is considerably stronger than that given in Figure 2 and produces a conclusive ranking for all those cases cited above, as well as many other previously ambiguous cases. The generalized Lorenz values, calculated as the product of the mean incomes and Lorenz values of Table 1, are reported in Table 2. Inspection of these figures indicates that the generalized Lorenz curves intersect in only 31 of the 190 potential pairwise comparisons. Thus, ordering the national distributions on the basis of generalized Lorenz curve dominance is conclusive in 84 per cent of the cases, three times the success rate obtained previously.

The resulting ordering over the 20 countries is illustrated in Figure 3. Not surprisingly, the ranking corresponds closely to that based on GDP per capita, ${ }^{12}$ and it is only those countries with broadly similar average income levels that provide examples of inconclusive rankings. In all other cases the differences in mean incomes is more than sufficient to offset any variations in the relative shares of the lower income groups in each of the countries. ${ }^{13}$

\section{Alternative Conceptions of Efficiency Preference}

While the welfare standard has been assumed to reflect a desire for both greater equality and higher incomes, ceteris paribus, it is not obvious what should be contained in the ceteris paribus clause. In the case of equity preference, the S-concavity condition concerns a reduction in inequality keeping the mean income constant. This suggests that the statement of efficiency preference should be related to an increase in incomes while maintaining the same degree of inequality. Yet this is clearly not the case under 
TABLE 2

Generalized lorenz Curve VAlues, $G L(y, p)^{*}$

\begin{tabular}{|c|c|c|c|c|c|c|c|c|c|c|c|}
\hline$p=$ & $10 \%$ & $20 \%$ & $30 \%$ & $40 \%$ & $50 \%$ & $60 \%$ & $70 \%$ & $80 \%$ & $90 \%$ & $95 \%$ & 100 \\
\hline ra & & & & & 2 & & & 7 & 1 & & \\
\hline bia & & & & $1 \cdot 8$ & $2 \cdot 6$ & & 5 & $7 \cdot 1$ & $9 \cdot 7$ & 1 & $7 \cdot 5$ \\
\hline ark & $1 \cdot 2$ & $4 \cdot 0$ & $7 \cdot 8$ & $12 \cdot 4$ & $18 \cdot 0$ & $24 \cdot 7$ & $32 \cdot 7$ & $42 \cdot 4$ & $54 \cdot 7$ & $63 \cdot 1$ & $73 \cdot 4$ \\
\hline ane & $0 \cdot 3$ & $1 \cdot 7$ & 4. & $7 \cdot 4$ & $11 \cdot 5$ & $16 \cdot 7$ & $23 \cdot 1$ & $31 \cdot 3$ & $42 \cdot 3$ & $49 \cdot 9$ & $63 \cdot 1$ \\
\hline & 0. & 0 . & 0 . & $0 \cdot 9$ & 1. & 1 & 2. & ? & $\cdot 7$ & .2 & $6 \cdot 1$ \\
\hline & & 0 & & 9 & & & 0 & $\cdot 5$ & $\cdot 0$ & $\cdot 4$ & $5 \cdot 1$ \\
\hline & & & & $12 \cdot 1$ & $16 \cdot 6$ & 21.9 & $27 \cdot 9$ & $35 \cdot 0$ & $43 \cdot 7$ & $49 \cdot 2$ & $57 \cdot 7$ \\
\hline & & & & & & & & & J & & $7 \cdot 3$ \\
\hline & & & & & $3 \cdot 0$ & & 5 & 7.8 & $10 \cdot 5$ & $2 \cdot 5$ & $17 \cdot 3$ \\
\hline & $0 \cdot($ & 2 & 5. & $8 \cdot 5$ & $12 \cdot 6$ & $17 \cdot 7$ & 23. & $31 \cdot 6$ & 41 & & $62 \cdot 3$ \\
\hline d & 0 . & $3 \cdot 2$ & $6 \cdot 6$ & $10 \cdot 8$ & $15 \cdot 8$ & 21 . & $28 \cdot$ & $37 \cdot 4$ & $48 \cdot 1$ & $54 \cdot 7$ & $64 \cdot 6$ \\
\hline & $0 \cdot 5$ & $3 \cdot$ & $7 \cdot 0$ & $11 \cdot 7$ & $17 \cdot 2$ & $23 \cdot 7$ & $31 \cdot 4$ & $40 \cdot 4$ & $51 \cdot 3$ & $58 \cdot 1$ & $68 \cdot 4$ \\
\hline & $0 \cdot 3$ & 1 . & 2 . & 3 & $5 \cdot 7$ & $8 \cdot 0$ & $10 \cdot 7$ & $14 \cdot 1$ & $18 \cdot 7$ & $21 \cdot 7$ & $27 \cdot 9$ \\
\hline $\mathrm{ka}$ & $0 \cdot 2$ & 0 . & 0.9 & $1 \cdot 5$ & $2 \cdot 1$ & $2 \cdot 9$ & 3 . & $\cdot 1$ & $\cdot 7$ & $\cdot 8$ & $9 \cdot 5$ \\
\hline & $1 \cdot 3$ & 4 . & $8^{\cdot}$ & $14 \cdot 0$ & $20 \cdot 4$ & $28 \cdot 1$ & $37 \cdot 2$ & $48 \cdot 4$ & $62 \cdot 8$ & $72 \cdot 2$ & $86 \cdot 6$ \\
\hline & & & & & & & $1 \cdot 6$ & $2 \cdot 1$ & $2 \cdot 8$ & $\cdot 3$ & $5 \cdot 3$ \\
\hline & & & & & & & 4 & & $\cdot 6$ & .5 & $13 \cdot 7$ \\
\hline & $1 \cdot$ & & 7 & $11 \cdot 4$ & $16 \cdot 3$ & 21 . & 28 & $35 \cdot 7$ & $5 \cdot 0$ & & $62 \cdot 5$ \\
\hline & 0. & & & & & & $11 \cdot 9$ & $16 \cdot 6$ & $23 \cdot 3$ & & $35 \cdot 6$ \\
\hline West Germany & $1 \cdot 5$ & $4 \cdot 2$ & $7 \cdot 5$ & $11 \cdot 5$ & $16 \cdot 2$ & $21 \cdot 5$ & $27 \cdot 6$ & $34 \cdot 9$ & $44 \cdot 0$ & 49. & $74 \cdot 5$ \\
\hline
\end{tabular}

* Computed as mean income $\times$ Lorenz curve value, or, equivalently, as $p \times$ mean income of lowest $p$ per cent of income recipients. All income values expressed as proportion of 1970 USA GDP per capita.

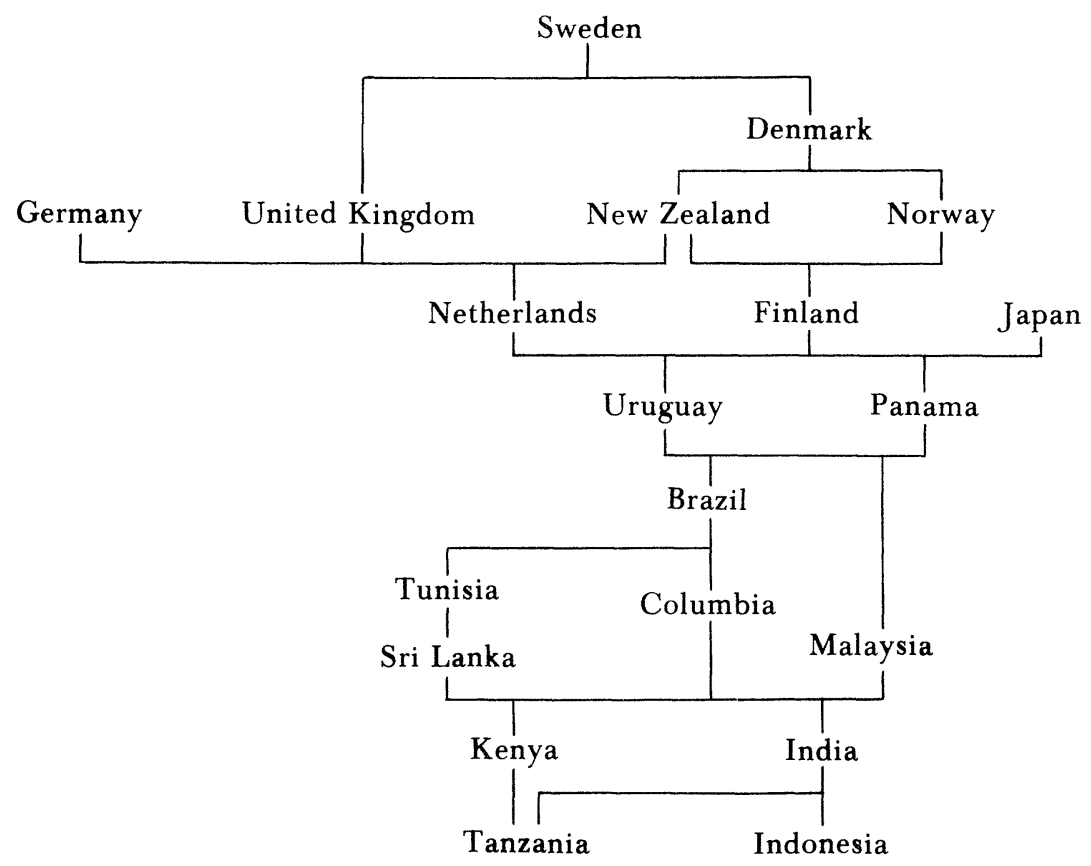

FIGURE 3. Generalized Lorenz curve ranking. 
the natural interpretation given in (5), since there is no reason to expect that increments to one or more incomes will leave the inequality level unaltered. On the contrary, a large increase in the income of the richest person would be universally regarded as representing an increase in inequality. For this reason the statement of efficiency preference given in (5) may come into conflict with the social desire for more equal distributions.

In the case of utilitarian welfare functions, the potential conflict between efficiency gains resulting from income increments and the possible equity losses is always resolved on the side of the efficiency gain. So the monotonicity condition (5) is always satisfied. The same will be true of any welfare function that is "individualistic" (depending only on individual welfare levels), if individual welfare levels are independent of other people's incomes. But it may not apply if we wish to incorporate elements other than individual welfare levels into the welfare function, or if we recognize that a person's sense of wellbeing is not independent of the standards of his friends and neighbours. ${ }^{14}$ If income differences are a source of envy, or are socially divisive for some other reason, we cannot presume that an increase in the income of one individual will not have repercussions on the welfare levels of others that lead to an overall reduction in welfare. In these circumstances it seems questionable whether it should be treated as axiomatic that an increment to any persons income necessarily improves the standard of welfare, as is done in condition (5).

Two issues are relevant at this point. The first concerns whether the social evaluation of distributions should reflect the opinions of individuals if these are influenced by envy, malice, myopia or similar considerations. In these circumstances there are grounds for arguing that social decisions should override the feelings of individual members - that they should be based on how people ought to respond to situations, rather than how they actually react. Without discussing this issue in any depth (see, for example, Sen, 1973, p. 51), it is clear that the monotonicity conditions (5) might be justified by appealing to such arguments. The results of Theorem 2 would then continue to be the appropriate procedure for implementing the ordering of distributions.

However, if we reject this line of reasoning, and with it the monotonicity condition, a second issue arises: what alternative concept of efficiency preference is satisfactory, and how does this affect the distributional ranking? ${ }^{15}$ One possibility is the requirement that welfare improves if all incomes are increased in the same proportion.

$$
\text { (scale improvement) } W(k \mathbf{y}) \geq W(\mathbf{y}) \text { for all } k \geq 1
$$

Here the increase in efficiency associated with a higher value of $k$ maintains the same distribution of relative incomes. It therefore corresponds to a preference for higher incomes keeping relative inequality constant.

Condition (11) is weaker than that given in (5) and the associated partial ordering $\geqslant_{2}$, defined by

$$
\mathbf{y} \geqslant_{2} \mathbf{y}^{\prime} \quad \text { iff } W(\mathbf{y}) \geq W\left(\mathbf{y}^{\prime}\right) \text { for all } W(.) \in \mathscr{W}_{2},
$$

where $\mathscr{W}_{2}=\{W() \mid. W($.$) satisfies (2) and (11) \}$, is consequently weaker than the ordering $\geqslant_{1}$. In fact, it is easily demonstrated that $\geqslant_{2}$ is equivalent to 
the common practice of ranking distributions on the basis of their means and Lorenz curves.

Theorem 3

$$
\begin{aligned}
& W(\mathbf{y}) \geq W\left(\mathbf{y}^{\prime}\right) \text { for all } W(.) \in W_{2} \\
& \text { iff } \mu \geq \mu^{\prime} \text { and } L(\mathbf{y}, p) \geq L\left(\mathbf{y}^{\prime}, p\right) \text { for all } p .
\end{aligned}
$$

Proof. (if) Define $\mathbf{y}^{\prime \prime}=\mu \mathbf{y}^{\prime} / \mu^{\prime}$. Then $W\left(\mathbf{y}^{\prime \prime}\right) \geq W\left(\mathbf{y}^{\prime}\right)$. Furthermore, $\mu=\mu^{\prime \prime}$ and $L(\mathbf{y}, p) \geq L\left(\mathbf{y}^{\prime}, p\right)=L\left(\mathbf{y}^{\prime \prime}, p\right)$ for all $p$. Hence, by Lemma $1, W(\mathbf{y}) \geq W\left(\mathbf{y}^{\prime \prime}\right) \geq$ $W\left(\mathbf{y}^{\prime}\right)$ for all $W(.) \in \mathscr{W}_{2}$.

(only if) Suppose $W(\mathbf{y})=\mu^{\alpha} f(\mathbf{y} / \mu)$ where $\alpha \geq 0$ and $f($.$) is S-concave.$ Then $W(.) \in \mathscr{W}_{2}$ and $W(\mathbf{y}) \geq W\left(\mathbf{y}^{\prime}\right)$ implies

$$
\mu^{\alpha} f(\mathbf{y} / \mu) \geq \mu^{\prime \alpha} f\left(\mathbf{y}^{\prime} / \mu^{\prime}\right)
$$

By choosing $f(\mathbf{y} / \mu) \equiv 1$, we have $\mu \geq \mu^{\prime}$. Alternatively, by choosing $\alpha=0$, we have $f(\mathbf{y} / \mu) \geq f\left(\mathbf{y}^{\prime} / \mu^{\prime}\right)$. But $f($.$) is an arbitrary S-concave function and$ $\mathbf{y} / \mu, \mathbf{y}^{\prime} / \mu^{\prime}$ have the same mean $(=1)$. Hence by Lemma 1 ,

$$
L(\mathbf{y}, p)=L(\mathbf{y} / \mu, p) \geq L\left(\mathbf{y}^{\prime} / \mu^{\prime}, p\right)=L\left(\mathbf{y}^{\prime}, p\right) \quad \text { for all } p
$$

Theorem 3 provides the welfare rationalization of the standard procedure of ranking one distribution above another if and only if it has a higher mean and a higher Lorenz curve. It also indicates the implications of replacing the "monotonicity" interpretation of efficiency preference with the weaker one of "scale improvement". As we have seen in the previous section, the welfare dominance relation $\geqslant_{2}$ is considerably weaker than $\geqslant_{1}$, and it will be likely that many, if not most, pairwise comparisons of distributions will be inconclusive. But even the "scale improvement" interpretation of efficiency preference may be too strong to be universally acceptable. For while scaling up the distribution leaves relative incomes unchanged, absolute differences between incomes are widened. Those for whom these absolute differences are important (for example, subscribers to Kolm's (1976a, b) "leftist" inequality measure) may well reject (11) on the grounds that the increase in efficiency may not offset the rise in inequality.

Another variant of efficiency preference that is sufficiently weak to cause no offence involves fixed additions to each person's income:

$$
\text { (incremental improvement) } W(\mathbf{y}+\alpha \mathbf{e}) \geq W(\mathbf{y}) \text { for all } \alpha \geq 0,
$$

where $\mathbf{e}$ is the unit vector $(1,1, \ldots, 1)$. Here the rise in mean income maintains the same absolute differences between incomes and reduces relative differences. There would not therefore seem to be any increase in inequality to offset the efficiency gain from higher incomes, so the efficiency and equity preferences of society do not come into conflict.

Unfortunately, a universally acceptable concept of efficiency preference is achieved only by a further weakening of the corresponding partial ordering over distributions. Letting $\mathscr{W}_{3}=\{W() \mid. W($.$) satisfies (2) and (13) \}$, we have $\mathscr{W}_{2} \subset \mathscr{W}_{3}$, since an incremental improvement to all incomes can be achieved by first raising all incomes proportionately and then performing mean- 
preserving progresssive transfers. Consequently, the ordering $\geqslant_{3}$ induced by $\mathscr{W}_{3}$ :

$$
\mathbf{y} \geqslant_{3} \mathbf{y}^{\prime} \quad \text { iff } W(\mathbf{y}) \geq W\left(\mathbf{y}^{\prime}\right) \quad \text { for all } W(.) \in \mathscr{W}_{3}
$$

is weaker than the ordering induced by $\mathscr{W}_{2}$. To assess just how much weaker the ranking will be, it is again necessary to obtain equivalent conditions that could be implemented empirically.

\section{Theorem 4}

$$
\begin{aligned}
& W(\mathbf{y}) \geq W\left(\mathbf{y}^{\prime}\right) \text { for all } W(.) \in \mathscr{W}_{3} \\
& \text { iff } G L(\mathbf{y}, p)-G L\left(\mathbf{y}^{\prime}, p\right) \geq\left(\mu-\mu^{\prime}\right) p \geq 0 \text { for all } p .
\end{aligned}
$$

Proof. (if) Define $\mathbf{y}^{\prime \prime}=\mathbf{y}^{\prime}+\left(\mu-\mu^{\prime}\right)$ e. Then $W\left(\mathbf{y}^{\prime \prime}\right) \geq W\left(\mathbf{y}^{\prime}\right)$. Furthermore, $\mu=\mu^{\prime \prime}$ and $G L\left(\mathbf{y}^{\prime \prime}, p\right)=G L\left(\mathbf{y}^{\prime}, p\right)+p\left(\mu-\mu^{\prime}\right) \leq G L(\mathbf{y}, p)$ for all $p$. Thus $L\left(\mathbf{y}^{\prime \prime}, p\right) \leq L(\mathbf{y}, p)$ and, by Lemma $1, W(\mathbf{y}) \geq W\left(\mathbf{y}^{\prime \prime}\right) \geq W\left(\mathbf{y}^{\prime}\right)$.

(only if) Suppose $W(\mathbf{y})=\mu^{\alpha} f\{\mathbf{y}+(\lambda-\mu)$ e $\}$ where $\alpha \geq 0, \lambda>0$ and $f$ is S-concave. Then $W(.) \in \mathscr{W}_{3}$. By choosing $f(\mathbf{y}) \equiv 1$, we have that $W(\mathbf{y}) \geq W\left(\mathbf{y}^{\prime}\right)$ for all $W(.) \in \mathscr{W}_{3}$ implies $\mu \geq \mu^{\prime}$. Alternatively, by choosing $\alpha=0$, we obtain $f\{\mathbf{y}+(\lambda-\mu) \mathbf{e}\} \geq f\left\{\mathbf{y}^{\prime}+\left(\lambda-\mu^{\prime}\right) \mathbf{e}\right\}$ for any S-concave function $f$. But $\mathbf{y}+(\lambda-\mu) \mathbf{e}$ and $\mathbf{y}^{\prime}+\left(\lambda-\mu^{\prime}\right)$ e have the same mean $(=\lambda>0)$. Hence, by Lemma 1 ,

$$
L\{\mathbf{y}+(\lambda-\mu) \mathbf{e}, p\} \geq L\left\{\mathbf{y}^{\prime}+\left(\lambda-\mu^{\prime}\right) \mathbf{e}, p\right\} \text { for all } p .
$$

So

$$
G L\{\mathbf{y}+(\lambda-\mu) \mathbf{e}, p\} \geq G L\left\{\mathbf{y}^{\prime}+\left(\lambda-\mu^{\prime}\right) \mathbf{e}, p\right\} \text { for all } p
$$

which, by the definition of $G L(\mathbf{y}, p)$, is equivalent to

$$
G L(\mathbf{y}, p)+(\lambda-\mu) p \geq G L\left(\mathbf{y}^{\prime}, p\right)+\left(\lambda-\mu^{\prime}\right) p \quad \text { for all } p .
$$

Condition (15) can be used to test whether any pair of distributions $\mathbf{y}, \mathbf{y}^{\prime}$ are ranked under the ordering $\geqslant_{3}$. One method of doing so is to calculate $G L(\mathbf{y}, p)-\mu p$ for each of the distributions and make pairwise comparisons of these values and the mean incomes. However, it will normally be sufficient to look only at the lowest income group in each of the populations. For the condition that

$$
G L(\mathbf{y}, p)-\mu p \geq G L\left(\mathbf{y}^{\prime}, p\right)-\mu^{\prime} p \quad \text { for all } p^{16}
$$

is equivalent to

$$
L(\mathbf{y}, p) \geq \frac{\mu^{\prime}}{\mu} L\left(\mathbf{y}^{\prime}, p\right)+\left(1-\frac{\mu^{\prime}}{\mu}\right) p \quad \text { for all } p
$$

and this imposes a high minimum Lorenz value constraint at the lower end of the distribution if $\mu$ is substantially higher than $\mu^{\prime}$. Thus if $\mu \geq 2 \mu^{\prime}$, it is necessary that $L(y, .1) \geq 5$ per cent in order that $\mathbf{y} \geqslant_{3} \mathbf{y}^{\prime}$, and such a value is rarely achieved in practice. Therefore, it seems empirically unlikely that distributions with significantly different means will be ranked under the ordering $\geqslant_{3}{ }^{17}$

This prediction is confirmed by the inter-country data examined in the previous section. Of the 53 conclusive pairwise comparisons under the ordering $\geqslant_{2}$, only 2 survive the more stringent conditions imposed by the ordering 
$\geqslant_{3}$ : the United Kingdom is still ranked above the Netherlands, and New Zealand above Finland. Both of these cases involve countries with very similar mean incomes. That the ordering $\geqslant_{3}$ manages to achieve conclusive results in only 2 of the 190 potential pairwise comparisons between countries makes it almost useless for practical purposes: if we are only prepared to impose restrictions (2) and (13) on the welfare function, we can expect little guidance on the relative desirability of different distributions.

\section{CONCLUSIONS}

This paper has examined the problem of ranking income distributions using a social welfare function $W($.$) . In particular, we have investigated$ whether it is possible to impose restrictions on $W($.$) that are both sufficiently$ weak to command a wide degree of support, and sufficiently strong to produce a conclusive ranking in many practical situations. The assumption that $W($. is equity-preferring (in the sense of being Schur-concave) and a non-decreasing function of all incomes (one interpretation of "efficiency preference") seems to achieve this dual objective. Furthermore, the corresponding ranking can be easily implemented by seeking a dominance relation between "generalized Lorenz curves", obtained by scaling up the ordinary Lorenz curve by the mean of the distribution. When applied to a comparison between income distributions in 20 countries, this ordering produced conclusive results in 84 per cent of the pairwise comparisons.

It can, however, be argued that the assumption that $W($.$) is increasing in$ all incomes is unacceptable, since it may be in conflict with the desire for greater equality. Replacing this with the weaker assumption that society values a proportional improvement in all incomes generates an ordering equivalent to the common practice of ranking distributions only when one has both a higher mean and higher Lorenz curve. But this may also be criticized on the grounds that a proportional improvement in all incomes increases absolute income differences. Substituting the even weaker assumption, that a constant absolute increase in everyone's income raises welfare, results in a ranking that is probably uncontroversial, but at the same time is too weak to be empirically useful.

\section{ACKNOWLEDGMENTS}

Some of the results in the paper were first presented at a symposium at Stanford University in August 1978. This version of the paper was discussed at the Public Finance Workshop at Queen's University, Ontario, during September 1981 and subsequently at an Economic Theory/Public Economics conference at Clare College, Cambridge. I am grateful to participants at these sessions, and to an anonymous referee, for their helpful comments. The study was undertaken in connection with a project on income distribution and income dynamics financed by the SSRC.

\section{NOTES}

\footnotetext{
${ }^{1}$ In practice, the question of comparability between households is typically examined independently, and adjustments to compensate for different household characteristics are applied to nominal incomes before considering the issue of ranking distributions (see, for example, Willig, 1981). So the assumption of a homogeneous population of households is not as serious a restriction as it may appear at first sight.
} 
2 The "welfare standard" examined in this paper is somewhat similar to the concept of "real national income" considered by Sen (1976). (See also Sen (1979a) and the exchange between Usher (1980) and Sen (1980).) However, we bypass most of the fundamental issues discussed by Sen (1976) and concentrate instead on the distributional aggregation question that he examines briefly in Section 8 .

${ }^{3}$ In the words of Sen (1973, p. 61), "The problem of extending the Lorenz partial ordering to cases of variable mean income is quite a serious one and this . . . restricts severely the usefulness of this approach."

${ }_{5}^{4}$ For simplicity it is assumed that all distributions under consideration have positive means.

${ }^{5}$ See also Kolm (1976b), Section VIII. Kolm's “concentration curves" are simple transformations of the generalized Lorenz curves defined here.

${ }^{6}$ The curves drawn correspond to the data for Indonesia and Malaysia provided in Tables 1 and 2 below. Note that in any pairwise comparison the generalized Lorenz curve for one distribution can be made identical to its ordinary Lorenz curve by a suitable choice of the vertical scale. Note also that each generalized Lorenz curve will have its own "diagonal of complete equality". This enables the representation of the Gini coefficient as the area above the (generalized) Lorenz curve relative to the area below the (mean-specific) "diagonal of complete equality" to be retained.

${ }^{7}$ The converse of this corollary is also true and follows immediately from the results of Marshall and Olkin (1979, p. 10). In their terminology, condition (9) is equivalent to the statement that $\mathbf{y}$ is "weakly supermajorized" or "weakly majorized from above" by $\mathbf{y}$ '.

${ }^{8}$ Of course, the term "higher" allows the possibility that the means are identical and that the Lorenz curve ordinates are equal at some or all points. For one distribution to be strictly preferred to another, we can take the anti-symmetric part of the relation $\geqslant_{1}$ and define $\mathbf{y}>_{1} \mathbf{y}^{\prime}$ if $\mathbf{y} \geqslant_{1} \mathbf{y}^{\prime}$ and not $\mathbf{y}^{\prime} \geqslant_{1} \mathbf{y}$. This would mean that all $W\left(\right.$.) rank $\mathbf{y}$ at least as good as $\mathbf{y}^{\prime}$ and some $W($.$) rank \mathbf{y}$ as strictly better than $\mathbf{y}^{\prime}$. This will occur when (9) holds and $G L\left(\mathbf{y}, p^{*}\right)>G L\left(\mathbf{y}^{\prime}, p^{*}\right)$ for some $p^{*}$.

${ }^{9}$ See Sen (1976, p. 28). This is his Axiom of Size Independence applied to a single community.

${ }^{10}$ Comparisons of the curves are made only at the points for which data are available. Other intersections may, of course, take place within these ranges (e.g. within the lowest decile).

${ }^{11}$ This is a Hesse diagram, in which dominance is indicated by a connected line flowing downwards from the higher ranked country.

${ }^{12}$ The ranking is, of course, a subrelation of the ordering by per capita GDP, since the "distributionally indifferent" welfare function $W(\mathbf{y})=\mu$ is admissible and insists on this condition being met. There is no possibility, therefore, of the greater equality in one distribution being sufficient to overturn the ranking by mean income, although this will be true for particular forms of $W($.$) , in which case the ranking by \geqslant_{1}$ will become inconclusive.

${ }^{13}$ Lorenz curve comparisons have the advantage of being independent of the unit of account, and the problems of converting currencies or of adjusting for inflation over time do not arise. Generalized Lorenz curves do not have this property, and the inter-country comparison therefore requires a transformation to a common currency unit (which in our example is based on purchasing power parities). While we have used a unique conversion rate, which might be subject to error, it would still be possible to rank distributions if one were only prepared to state a range for the appropriate conversion rate.

${ }^{14}$ See, for example, Sen's criticisms of "welfarism" in Sen (1979b, Section VI), and elsewhere.

${ }^{15}$ Here we revert to a comparison of distributions over a population of constant size $n$. But the results are again easily extended to populations of different sizes.

${ }^{16}$ This rearrangement of (15) suggests how the condition can be interpreted geometrically. Not only must the generalized Lorenz curve $G L(\mathbf{y}, p)$ lie everywhere above $G L\left(\mathbf{y}^{\prime}, p\right)$, but the vertical distance from its corresponding equality diagonal $(=\mu p-G L(\mathbf{y}, p))$ must also be everywhere smaller.

${ }^{17}$ Another useful analytical device is the profile of $\mu(p)$, defined as the mean income of the lowest $100 p$ per cent of income recipients. Thus

$$
\mu(p)=G L(\mathbf{y}, p) / p=\mu L(\mathbf{y}, p) / p
$$

and $\mu(1)=\mu$. The graph of this function represents a "cumulated mean income curve" which seems appropriate to abbreviate to COMIC. COMICs are non-decreasing functions of $p$, rising from $y_{1}$ (when $p=1 / n$ ) to $\mu$ (when $p=1$ ). COMICs drawn for two distributions enable an immediate comparison to be made from both the viewpoint of a Rawlsian (for whom only the left-hand end of the graph would be relevant) and that of someone who is indifferent to the distribution of any aggregate income (for whom only the right-hand end-points would be relevant). Furthermore, Theorems 2 and 4 are easily translated in terms of $\mu(p)$. In Theorem 2 the condition that $\mu(p) \geq \mu^{\prime}(p)$ replaces $G L(\mathbf{y}, p) \geq G L\left(\mathbf{y}^{\prime}, p\right)$ : in other words, one COMIC lies everywhere above the other. For Theorem 4 , condition (15) becomes

$$
\mu(p)-\mu^{\prime}(p) \geq \mu-\mu^{\prime} \geq 0 \text { for all } p
$$


which, since $\mu(1)=\mu$, means that the minimum distance between the two COMICs occurs at the right-hand end-point, corresponding to $p=1$.

\section{REFERENCES}

BLACKORBY, C. and DONALDSON, D. (1977). Utility vs equity: some plausible quasi-orderings. Journal of Public Economics, 7, 365-381.

DASGUPtA, P., SEN, A. K. and STARRETT, D. (1973). Notes on the measurement of inequality. Journal of Economic Theory, 6, 180-187.

JAIN, S., (1975). Size Distribution of Income. Washington: World Bank.

$\rightarrow$ KolM, S.-CH. (1976a). Unequal inequalities, I. Journal of Economic Theory, 12, 416-442.

- (1976b). Unequal inequalities, II. Journal of Economic Theory, 13, 82-111.

Kravis, I. B. Heston, A. W. and Summers, R. (1978). Real GDP per capita for more than one hundred countries. Economic Journal, 88, 215-242.

MARShAll, A. W. and OlKIn, I. (1979). Inequalities: Theory of Majorization and its Applications. New York: Academic Press.

RothSCHILD, M. and Stiglitz, J. E. (1973). Some further results on the measurement of inequality. Journal of Economic Theory, 6, 188-204.

SEN, A. K. (1973). On Economic Inequality. Oxford: Univeristy Press.

$\rightarrow$ (1976). Real national income. Review of Economic Studies, 43, 19-39.

- (1979a). The welfare basis of real income comparisons: a survey. Journal of Economic Literature, 17, 1-45.

(1979b). Personal utilities and public judgements: Or what's wrong with welfare economics. Economic Journal, 89, 537-558.

$\rightarrow \rightarrow(1980)$. The welfare basis of real income comparisons: a reply. Journal of Economic Literature, 18, $1547-1552$.

UsHeR, D. (1980). The welfare basis of real income comparisons: a comment. Journal of Economic Literature, 18, 1544-1547.

Willig, R. D. (1981). Social welfare dominance. American Economic Review, 71, 200-204. 